\title{
EVOLUCION Y ESTRUCTURA PSICOLINGÜÍSTICA DE LA FUNCIÓN POÉTICA DEL LENGUAJE
}

\author{
EVOLUTION AND STRUCTURE PSICOLINGÜÍSTICA OF THE POETIC FUNCTION \\ OF THE LANGUAGE
}

RAÚL GONZÁLEZ MOREYRA ${ }^{1}$

Universidad Nacional Mayor de San Marcos, Facultad de Psicología

\section{RESUMEN}

OBJETIVO: Explorar significativas para nuestros problemas serían las siguientes: a) El procesamiento global de la emisión como una unidad textual. b) El procesamiento de la equivalencias tipo II fónicas. c) El procesamiento de la equivalencias tipo II semánticas. d) El procesamiento de la equivalencias tipo I morfosintácticas. DISEÑO: Descriptivo comparativo de corte transversal. MATERIAL Y MÉTODOS: Se utilizó la técnica del «cloze» para explorar la lectura de textos poéticos (fábulas) en 200 niños entre los 7 y 16 años. Los completamientos fueron calificados siguiendo la caracterización de Jakobson y Lewin sobre la función poética como equivalencias fonológicas, semánticas y morfosintácticas. RESULTADOS: Todas las correlaciones son significativas estadísticamente con una $\mathrm{p}<0.01$, salvo la de métrica que tiene una $\mathrm{p}<0.05$. Las variables edad $\mathrm{y}$ rendimiento están altamente correlacionadas. El puntaje global que es la sumatoria de las cuatro dimensiones tiene un coeficiente «rho» de 0.90 que expresa la alta asociación entre ambas variables. CONCLUSIONES: Los resultados demuestran el carácter evolutivo de los desempeños de los niños con respecto a esta función y el paralelismo de su desarrollo con las etapas piagetana lógico-concreta y lógica-formal.

Palabras claves: Función poética del lenguaje, técnica de Cloze.

\begin{abstract}
OBJECTIVE: To explore significant for our problems they would be the following ones: a) The global processing of the emission like a textual unit. b) the processing of equivalences type II phonics. el the processing of semantic equivalences type II. d) the processing of morfosyntactic equivalences type I. DESIGN: Descriptive comparative of cross section. MATERIAL And METHODS: The technique of «cloze» was used to explore the poetic text reading (fables) in 200 children between the 7 and 16 years. The completion were described following the characterization as Jakobson and Levin on the poetic function like phonological, semantic and morfo-syntactic equivalences. RESULTS: All the correlations are significant statistically with one $p<0,01$, except for the one of metric that has one $p<$ 0.05 . The variables age and yield highly are correlated. The global score that is additions of the four dimensions has a coefficient «rho» of 0,90 that expresses the high association between both variables. CONCLUSIONS: The results demonstrate the evolutionary character of the performances of the children with respect to this function and the parallel ism of its development with the stages piagetana logical-makes specific and logic-formal.
\end{abstract}

Keywords: Poetic function of the language, technique of Cloze.

${ }^{1}$ Publicación postuma 


\section{INTRODUCCIÓN}

En 1960 Jakobson $^{1}$ definía la función poética del lenguaje como aquella orientada hacia el mensaje proyectando el principio de equivalencia del eje de selección (paradigmático) al eje de combinación (sintagmático). Esta función se manifiesta en todo lenguaje, pero dominantemente en el lenguaje de la poesía con su reiteración regular de unidades equivalentes. Jakobson1 cita a Hopkins quien define el verso como un «discurso que en parte o totalmente repite una misma figura fónica». Las otras funciones comunicacionales que son identificadas por Jakobson1 en el mismo texto son las fática, emotiva, referencial, conativa y metalingüística.

También en 1960, Martinet ${ }^{2}$ definió la lengua como un instrumento comunicacional organizado en dos planos articulatorios. Una primera articulación: los monomas (morfemas) dotados de un contenido semántico y una expresión fónica; y una segunda articulación, en la que el componente fónico de la primera se articula a su vez en las unidades distintivas, sucesivas y de número finito para cada lengua que son los fonemas.

En 1962, Levin ${ }^{2}$ formaliza la propuesta de jakobson ${ }^{1}$ identificando dos tipos de equivalencia: 1) Tipo I, en él que la equivalencia está definida por la posición que la palabra puede ocupar en la oración (por ejemplo, todas las palabras que pueden ser núcleo de sujeto); 2) Tipo II, cuando las palabras tienen la misma referencia, como en el caso de los sinónimos, o cuando tienen propiedades fónicas similares, como igual rima o metro. La naturaleza de la poesía es la utilización de equivalencias tipo II, es decir, clases con contorno romántico o fónico común. Debe notarse que, según el concepto de la lengua de Martinet $^{3}$ las equivalencias propuestas por Levin ${ }^{2}$ como las de carácter poético corresponderían a las dos articulaciones en forma diferenciada: las equivalencias semánticas corresponderían a la primera articulación y las equivalencias fónicas a la segunda. Nosotros, en publicaciones y exposiciones anteriores (González, 1982), hemos argumentado que las funciones comunicacionales obedecen, desde la perspectiva psicológica, a un proceso evolutivo en la edad infantil que sigue en lo fundamental a los grandes periodos psicogenéticos de la inteligencia propuestos por Piaget (1947) ${ }^{4}$.

El periodo de la inteligencia sensorio-motriz de cero a dos años correspondería al despliegue de la función fática (Malinowski, 1923) ${ }^{5}$, es decir, al uso del lenguaje básicamente como apertura y mantenimiento del contacto interpersonal. Los trabajos de Luria $(1979)^{6}$ sobre el papel de la palabra en la economía comunicacional del lactante insertada sincréticamente en el complejo posicional, gestual y también objetal (tanto en el papel de la elección dinámica como de indicio) apuntan en esa dirección.

Las funciones emotiva, conativa y referencial de jakobson ${ }^{1}$ se desplegarían en el período de la inteligencia pre-operatoria de los dos a los siete años. En el modelo del «organon» del lenguaje de Bühler (1934)7 corresponden a las tres funciones de sentido: expresivas, apelativas y representacionales; es por ello que a este complejo funcional semantológica se ha ido formando en el período sensorio-motor pero se despliega y se hace dominante en la comunicación entre los dos y siete años.

La función metalingüística, orientada hacia el código que el hablante maneja intencionalmente, se desarrolla como función desplegada recién entre los siete años y doce años. Los indicadores psicológicos son muy claros: las definiciones léxicas recién se hacen posible hacia los siete años (Terman y Merrill, 1937)8; la efectiva madurez para el aprendizaje de la lectura requiere de una edad mental por encima de los siete años (Mialaret ${ }^{9}$, 1969), y aprender a leer es poner en correspondencia un código fónico con un código gráfico; es decir, una actividad esencialmente metalingüística. Por último, la enseñanza de la gramática recién es posible iniciarla -y muy cuidadosamente en la escuela a preadolescentes (Titone ${ }^{10}, 1976$ ), cuando la función metalingüística, es decir, la capacidad 
de análisis del propio código, ha madurado totalmente. Este período cubre la etapa del pensamiento lógico-concreto.

La función poética se centra en el mensaje y se expresa dominantemente en el juego lingüístico de la equivalencias; pero es obvio que será el aspecto principal de lo que Delas y Filliolet $(1973)^{11}$ llaman la «poesía constituida», formando el núcleo de esa totalidad en funcionamiento que configura un material textual unitario, como es un poema.

Nosotros creemos que la función poética, tal como la definimos arriba, se prepara en las edades que hemos ya descrito, pero que efectivamente se despliega en la fase del pensamiento lógico-formal entre los doce y quince años de edad, diferenciándose de lo «literario», concepto probablemente más abarcativo y que está ligado tanto a las estructuras de contenido como a otros aspectos semióticos de la creatividad verbal (Delas y Filliolet, $1973)^{11}$, tales como las dimensiones estática y estilística, por ejemplo.

En la investigación sobre creatividad infantil en el lenguaje generalmente se ha opuesto lo poético a lo prosaico, como polaridad entre el lenguaje respectivamente con fantasía afectiva y lenguajes banales (Freinet, 1974) ${ }^{12}$. Más recientemente, Desrosiers $(1978)^{13}$ ha hecho un excelente trabajo de análisis de contenido retórico en composiciones infantiles entre los ocho y once años como medida de creatividad. Sus resultados otorgan un promedio de 8.5 puntos para los cuatro grupos de edad sin diferencias significativas entre ellos. Nuestro concepto de lo poético no tiene vinculación con el de Freinet ${ }^{12}$, sino que se fundamenta en Jakobson ${ }^{1}$ - Levin ${ }^{2}$, ya diferencia de los resultados de Desrosiers ${ }^{13}$, que no presenta rasgos evolutivos en la edad infantil, nosotros pensamos que la función poética sí es evolutiva y precisamente en la etapa estudiada de los ocho a once años en el aspecto de creatividad literaria. Creemos que este desarrollo es significativo por preparar el advenimiento de la función poética desplegada hacia la pubertad y que esta evolución podría detectarse con procedimientos adecuados de exploración.

\section{Planteamiento del problema e hipótesis}

De la exposición de las propuestas lingüísticas sobre el carácter de la función poética y de las investigaciones sobre la creatividad verbal en los niños se desprende un divorcio entre ambas corrientes de investigación, la lingüístico-formal y la psicológico-comportamental. Nuestros problemas se derivan de este divorcio y se orientan a explorar una posible articulación lingüística, poética y psicológica en una psicolingüística poética que responda a dos preguntas fundamentales ¿Corresponde a la función poética diseñada por Jakobson ${ }^{1}$ una realidad psicológica? ¿Es evolutiva esta realidad psicológica? En otras palabras ¿Puede psicológicamente hablarse de una función poética como hablamos de una función perceptiva o motriz? y si podemos hacer esto, ¿Se despliega tal función en el tiempo en un proceso constructivo o tiene una estructura puramente sincrónica de la edad evolutiva, como parecen apuntar los resultados de Desrosiers ${ }^{13}$ ?

Desde el punto de vista psicolingüístico, una poesía puede ser considerada como una emisión que el receptor (lector) debe procesar. En nuestro caso las dimensiones a explorar significativas para nuestros problemas serían las siguientes:

1. El procesamiento global de la emisión como una unidad textual.

2. El procesamiento de la equivalencias tipo II fónicas.

3. El procesamiento de las equivalencias tipo II semánticas.

4. El procesamiento de la equivalencias tipo I morfo-sintácticas. 
Estas dimensiones deben obedecer a dos propiedades fundamentales que son nuestras hipótesis principales:

1. Deben evolucionar incrementándose con diferencias significativas en la edad evolutiva

2. Deben poseer una estructura psicológica subyacente que exprese el papel que cumplen las equivalencias tipo II fónicas y semánticas.

\section{MATERIALES Y MÉTODOS}

Se decidió la utilización del procedimiento de «Cloze» o cerramiento propuesto inicialmente por Taylor (1953)14 e incorporado a la investigación psicolingüistica por Wilson y Carrel $(1954)^{15}$. Consiste en suprimir del texto propuesto algunas unidades, en nuestro caso léxicas, de tal manera que el lector debe complementarias. Se presume (y toda la investigación existente lo confirma) que productor y receptor de un mensaje tienen hábitos semánticos y gramaticales similares, por tanto este último debe anticipar con alguna exactitud lo que producirá el emisor en algún momento de un mensaje bien estructurado. Es obvio que al someter a la técnica de «Cloze» un texto poético estamos explorando las anticipaciones de lectura poética del sujeto; en consecuencia las propiedades de su cerramiento serán indicadores de las propiedades de su función poética dinamizadas por la lectura que ejecutan los sujetos.

Se seleccionaron cuatro posesias (fábulas) cortas de un libro de lectura de educación básica (cuarto grado) de Aguayo1\&, a saber, "La zorra y las uvas» de Samaniego; «El jumento murmurador» de Glein; "El congreso de los ratones» de Lope de Vega; y «La zorra» de Lessing. Se dieron por escrito sin título, pero separadas por los rótulos Texto 1, Texto 2, etc. Cada poesía tuvo el siguiente tratamiento para la supresión léxica: se conservó siempre intacta la estrofa inicial que mostraba la estructura métrica y la combinación de rimas. Y se suprimió la mitad de palabras terminales de verso de cada una de las otras est ofas de cada poesía. Es decir, el sujeto recibía del modelo poético básico y debía completar palabras de final de verso. La consigna en todos los casos fue:

«Aquí hay unas poesías con unas rayas en donde falta una sola palabra, tu debes poner en esa raya la palabra que te parezca más adecuada». Los sujetos trabajaban libremente y se les pedía que solicitaran la ayuda que quisieran; se cuidó en este caso que la ortografía de la escritura fuera la más adecuada al cerramiento elegido para facilitar la calificación futura. En las explicaciones a los sujetos se insistió en que no había respuestas buenas y malas sino que pusieran simplemente la palabra que creían estaba mejor allí. En total debían completarse quince palabras por cada sujeto.

Se seleccionó una muestra de doscientos niños hombres y mujeres de dos colegios particulares de clase media de un distrito residencial de Lima. Los niños tenían de 7 a 16 años (10 por cada edad - sexo). Se exigió que tuvieran escolaridad normal y que no presentaran ningún rasgo patológico en las áreas afectivas y de lenguaje. Todos los niños debían ser hispanohablante, de hogares y madres del mismo tipo.

La calificación se realizó de la siguiente manera considerando cada grupo de edad - sexo como una unidad:

1. Medida de redundancia. Se explicó a cada grupo la fórmula de redundancia informacional lexica propuesta por McLeod y Anderson (1970) ${ }^{17}$. Esta medida proporciona la legibilidad que para cada sujeto tiene el texto en función de su grupo de 
pertenencia lectora. Es un indicador del procesamiento global del texto que no se ha utilizado en este informe.

2. Medida de las rimas. Se analizó cada completamiento desde el punto de vista de su composición fonológica a partir en vocales y consonantes (rima asonantada o rima aconsonanatada respectivamente) se le daba valor de 1; en caso contrario, valor cero. Este es un indicador de las equivalencias fónicas tipo II.

3. Medida de la métrica. Se contrastó en cada completamiento el número de sílabas y la acentuación de la palabra suprimida con la palabra colocada. Si había similaridad métrica, es decir, si la combinación de acentuación y de número de sílabas que exige la versificación española entre ambas palabras era la misma, se calificaba con 1; en caso contrario, con cero. Esta es también una equivalencia fónica tipo II. Debe anotarse que métricamente son palabras equivalentes a final de verso las siguientes parejas: página libre, árbol - sol, perro - tienda, etc.

4. Medida semántica. Las palabras completadas que tuvieran alguna (cualquiera de las relaciones semánticas propuestas por Palmer ${ }^{16}$ 1976) con la palabra original -sinonimia, antonimia, homonimia- se calificaban con 1; sino, con cero. Este es un indicador de la equivalencia tipo II semántica.

5. Medida Morfológica. La identidad paradigmática de clase de palabra se calificaba con 1 punto, como cuando un sustantivo suprimido es llenado por otro sustantivo. En caso contrario, la puntuación era cero. Este es un indicador de las equivalencias tipo I. 


\section{RESULTADOS}

En el cuadro $\mathrm{N}^{\circ} 1$ presentamos las puntuaciones promedio correspondientes a cada una de las variables medidas. Como puede apreciarse en la columna «rho» todas las correlaciones son significativas estadísticamente con la $\mathrm{p}<0.01$, salvo la de métrica que tiene una $\mathrm{p}<$ 0.05. Las variables edad y rendimiento están altamente correlacionadas. Obsérvese que el puntaje global que es la sumatoria de las cuatro dimensiones tiene un coeficiente «rho» de 0.90 que expresa la alta asociación entre ambas variables.

\section{Cuadro $\mathrm{N}^{\circ}$ 1: Puntuaciones promedio y coeficientes «rho» de los grupos de edad en las dimensiones medidas}

\begin{tabular}{|c|c|c|c|c|c|c|c|c|c|c|}
\hline \multicolumn{11}{|l|}{ Equivalencias } \\
\hline Edad & 7 & 8 & 9 & 10 & 11 & 12 & $13 \quad 14$ & 15 & 16 & Rho \\
\hline Rima & .12 & .19 & .25 & .28 & .29 & .34 & $.25 \quad .48$ & .44 & .46 & $.84 * *$ \\
\hline Métrica & .49 & .47 & .57 & .58 & .62 & .61 & 64 & .64 & .60 & $.73^{*}$ \\
\hline Semántica & .19 & .16 & .36 & .39 & .52 & .49 & $.46 \quad .59$ & .57 & .56 & $.89 * *$ \\
\hline Morfosintáctica & .77 & .77 & .82 & .82 & .81 & .78 & $.81 \quad .84$ & .86 & .87 & $.79 * *$ \\
\hline Global & 1.56 & 1.581 & 1.99 & 2.07 & 2.24 & 2.23 & 2.082 .55 & 2.52 & 2.50 & $.90 * *$ \\
\hline
\end{tabular}

Al observar la evolución de las puntuaciones vemos que la puntuación global avanza hasta los 12 años, luego a los 13 retrocede hasta un nivel de 10 años, y por último a los 14, 15 y 16 años prácticamente se estabiliza. Este tipo de evolución cuantitativa de los puntajes globales nos sugiere la hipótesis siguiente: la curva real y la curva ajustada de sus puntajes tal como se representan en el gráfico $\mathrm{N}^{\circ} 1$ ocultan una diferencia evolutiva en dos etapas: una primera etapa entre los 7 y 12 años de incremento regular, y una segunda etapa de 12 a 16 años con un índice «décalage» y una posterior estabilización. En el gráfico $\mathrm{N}^{\circ} 2$ se muestran los resultados de dividir la puntuación global en dos curvas: de 7 a 12 años y de 12 a 16 años. Al realizar el ajuste de curvas por el método de los cuadrados mínimos encontramos el incremento regular previsto de 7 a 12 años. De 12 años a 16 no existe un incremento regular pues la curva «o〉 se refleja las características de «décalage» y estabilidad de la curva «a». 


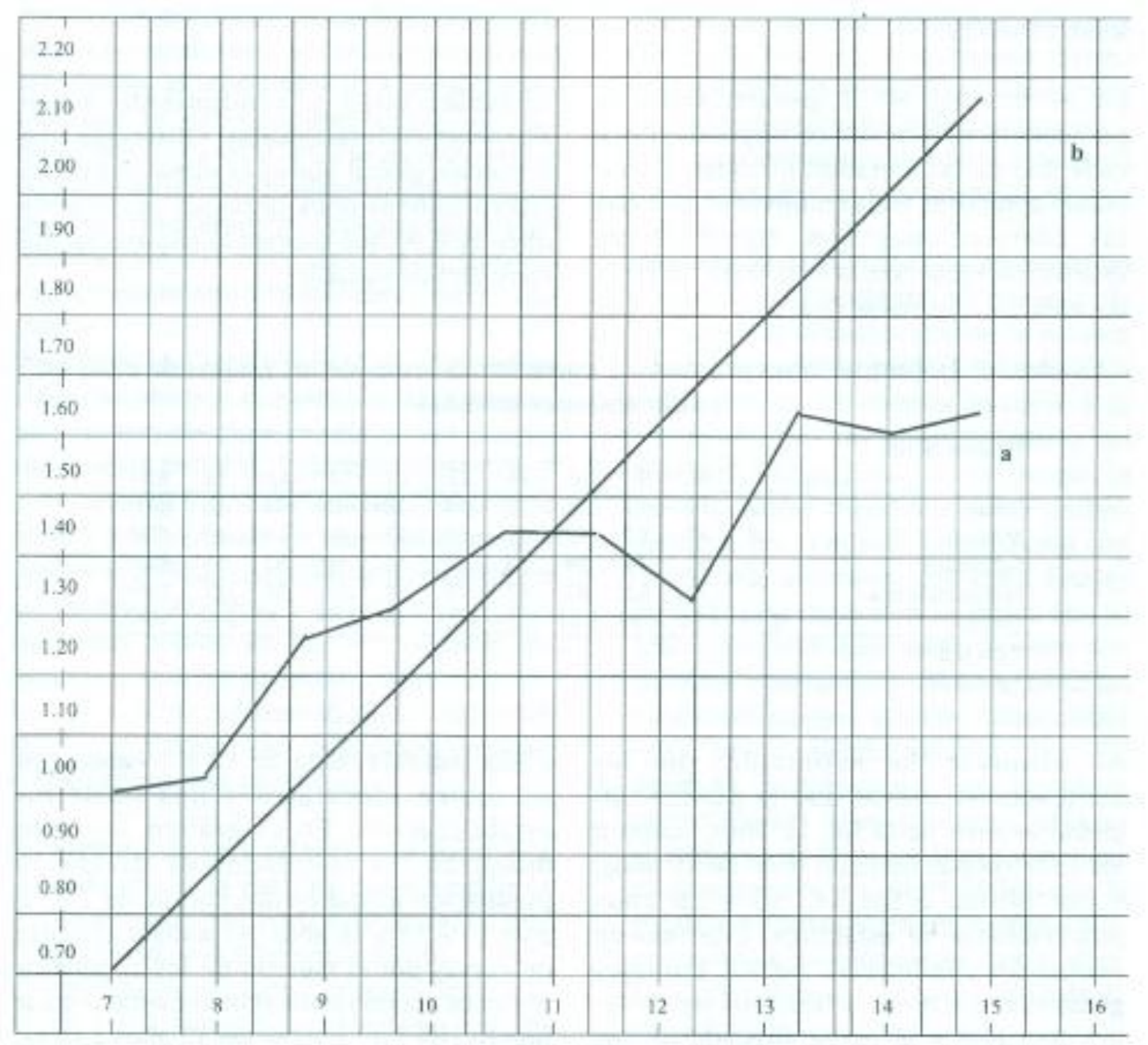

Gráfico $N^{\circ}$ 1.- Curva real y curva ajustada de las puntuaciones correspondientes a los 10 grupos de edad. 


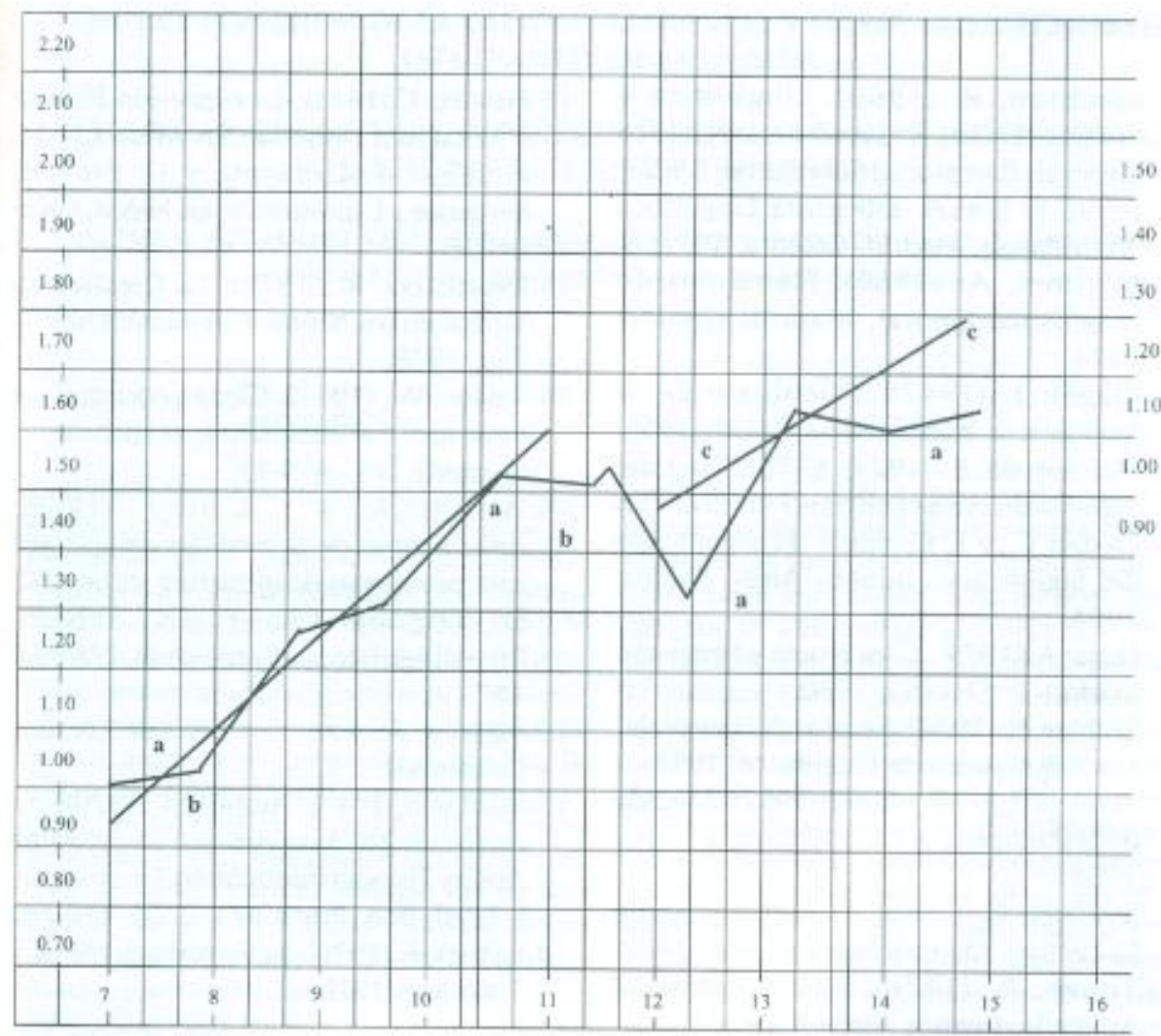

Gráfico $N^{\circ} 2$.- Curva real y curva ajustada de los grupos de edades: 7 - 12 años y 12 16 años.

\section{CONCLUSIONES}

Hemos explorado en una muestra de 200 niños de primaria y secundaria de Lima el completamiento lector de un grupo de fábulas infantiles. Se le aplico a los completamientos de una técnica de calificación que refleja las propiedades de la función poética tal como fuera descrita por Jakobson ${ }^{1}$ y Levin ${ }^{2}$; nuestros resultados demuestran:

1. El carácter evolutivo del completamiento explorado: los niños mejoran progresivamente la calidad del completamiento en función de los indicadores propuestos.

2.

3. El rendimiento tiene dos etapas: una primera de 7 a 12 años de incremento regular y una segunda, de 12 a 16 años con un breve «décalage» y su posterior estabilización, etapas paralelas a las propuestas por Piaget para la inteligencia operatoria. 


\section{REFERENCIAS}

1. Jakobson, R. (1960). Lingüística y Poética. En Id.: Ensayos de Lingüística General. Barcelona: Seix Barral, 1975.

2. Levin, S. (1962). Estructura Lingüística en la Poesía. Madrid: Cátedra, 1977.

3. Martinet, A. (1960). Elementos de Lingüística Genral. Madrid: Gredos, 1974

4. Piaget, J. (1947). Psicología de la Inteligencia. Buenos Aires: Piados, 1955.

5. Malinowski, B. (1923). El Problema del Significado en las Lenguas Primitivas. En Ogden C y 1. Richards: El Significado del Significado. Buenos Aires: Paidos, 1964

6. Luria, A. (1979). Conciencia y Lenguaje. Madrid: P. Del Río, 1980.

7. Bülhler, K. (1934). Teorías del Lenguaje. Madrid: Revista de Occidente. 1981.

8. Terman, L. y M. Merrill (1957). Medida de la Inteligencia. Madrid: Espasa Calpe, 1958.

9. Mialaret, G. (1969). El Aprendizaje de La Lectura. Madrid: Marova - Fax, 1972.

10.Titone, R. (1976). Psicolingüística Aplicada. Buenos Aires: Kapelusz.

11.Delas, D. y J. Filliolet (1973). Lingüística y Poética. Buenos Aires: Hachette, 1981.

12.Freinet, C(1973). La expresión Poética y la cultura personal. En Mesa L. ; M. Hidalgo; J. Urdaneta y C. Freinet: Lenguaje y Expresión en los Niños. Lima: INIDE.

13.Desrosiers, R. (1978). La Creatividad Verbal en los Niños. Barcelona: OikosTau, 1978.

14.Taylor, W. (1953). Cloze procedure: A new tool for measuring readability J. Quaterly, 30. 415-33.

15.Wilson, K. y J. Carrell. (1954). Aplicaciones de la medidas de entropía a los problemas de estructura secuencial. En Osgood Ch. Y Th. Sebeek: Psicolingüística. Barcelona: Planeta, 1974.

16.Aguayo, A. (s.f.). Libro 4to de Lectura, Guatemala: CCA.

17.MacLeod, J. y J. Anderson (1970). An Aproach to Assessment of Reading Ability Througt Information Transmission. J. Read, Boh. Primavera, 2, (2), 116-143

18. Palmer, F. (1976). La Semántica. México: Siglo XXI. 1978. 